\title{
Intraocular Surgery Following Penetrating Keratoplasty: The Risks and Advantages
}

\author{
L. A. FICKER, C. M. KIRKNESS, A. D. McG STEELE, N. S. C. RICE, \\ A. M. E. GILVARRY \\ London
}

\begin{abstract}
Summary
Graft survival has been evaluated for patients who underwent subsequent intraocular surgery (extra-capsular cataract surgery or trabeculectomy) between 1983 and 1989. The patients were different from the majority of keratoplasty patients as evidenced by the indications for keratoplasty; corneal perforation was the indication in $\mathbf{2 4 \%}$ of cases. Perforated and inflamed eyes were treated aggressively at the time of the acute event, including emergency keratoplasty and intensive topical steroids. Visco-elastic fluids were routinely used during secondary surgery and topical steroids were administered intensively post-operatively. The incidence of post-operative graft rejection was low (less than $14 \%$ ). Rejection episodes were diagnosed early, prior to the appearance of a Khodadoust line, and were treated aggressively with intensive topical steroids. Glaucoma which was not controlled by topical therapy was surgically managed by trabeculectomy in the first instance. If this failed, tube drainage was performed and long-term topical steroids were administered. The only risk factor identified was uncontrolled glaucoma, $P=0.1$. The probability of graft survival (at five years) was 0.83 after cataract surgery and 0.62 after trabeculectomy, but wide confidence limits indicate the difference is not significant.
\end{abstract}

The impact of intraocular surgery upon a preexisting penetrating keratoplasty $(\mathrm{PK})$ is pertinent to various sub-groups of patients with corneal disease. The most frequent procedures to be performed subsequently are cataract extraction and trabeculectomy.Endothelial cell loss is known to be accelerated by intra-ocular surgery. ${ }^{1}$ Expected graft survival may therefore be compromised by accelerated endothelial cell loss following such surgery. Glaucoma may also threaten graft survival. It may be a complication of keratoplasty, or previously controlled glaucoma may become uncontrolled after further intraocular surgery. ${ }^{2}$ The introduction of viscoelastic fluids, topical beta-blockade and tube drainage procedures ${ }^{3}$ represent therapeutic advances with respect to these complications.
For patients in whom corneal disease coexists with early cataract there are advantages in deferring cataract surgery in patients undergoing PK. Stable keratometry following PK should allow greater accuracy in calculating IOL power. Furthermore, when PK is performed as an emergency procedure for perforation or progressive microbial keratitis, deferred cataract surgery (in the absence of an intumescent lens) may reduce post-operative inflammation, hence reducing the risk of early graft rejection. ${ }^{4}$ Knowledge of the risks and advantages of combined compared with twostage procedures would be useful in planning the management of complicated cases such as these.

Glaucoma which is uncontrolled by topical therapy requires surgery. Trabeculectomy 
and tube drainage surgery have been performed in patients with a pre-existing corneal graft and it would be useful to identify any risk factor for graft survival which may be related to glaucoma surgery.

The effects upon graft survival of modern microsurgery for cataract and glaucoma have been reported infrequently. We therefore reviewed our experience in the Corneal Clinic at Moorfields Eye Hospital. Since 1983, ECCE (mostly with lens implantation) has been the routine surgical technique for cataract surgery and tube drainage was adopted in the management of failed trabeculectomy, in preference to cyclocryo-ablation. ${ }^{5}$ In addition, visco-elastic fluids were routinely used by this time for intraocular surgery in the presence of a graft.

\section{Patients and Methods}

Patients who underwent intraocular surgery after prior penetrating keratoplasty, between 1983 and 1989, were identified from the Corneal Clinic database. Twenty-five patients underwent extracapsular cataract extraction, 17 with additional lens implantation. Two further patients underwent secondary lens implantation following combined PK and ECCE. Twenty-nine patients underwent trabeculectomy after PK and five of these underwent tube drainage procedures following failed trabeculectomy.

\section{Extracapsular Cataract Extraction}

The mean post-PK interval was 73.3 months overall $(n=25)$ and 33.4 months for the subgroup which had IOL implantation $(n=17)$. The interval was shorter (9 months) for inflamed eyes; two intumescent lenses were removed within one month. The two secondary implants were inserted at a mean interval of 91 months. Removal of graft sutures had previously been performed in about half the cases. The most frequent indications for PK were keratoconus, interstitial keratitis and corneal perforation (Table I). There were 20 first, five second and two subsequent PKs. Trabeculectomy had previously been performed in four cases. Most (22) were central grafts, mean $7.6 \mathrm{~mm}$ diameter. Three were eccentric, adjacent to the limbus and measuring between $4 \mathrm{~mm}$ diameter and $9 \times 12 \mathrm{~mm}$. The post-operative steroid regime was initially $1-2$ hourly topical dexamethasone $0.1 \%$ and was reduced according to the clinical course. Complications, graft survival, refractive and visual outcomes were analysed.

\section{Trabeculectomy}

Trabeculectomy was performed after a mean post-PK interval of 7.8 months $(n=29)$, ten of which were within one month. The most frequent indications for grafting were keratitis and trauma as shown in Table I. Trabeculectomy alone was performed in 20 patients; nine others underwent additional surgery; tube drainage (5), ECCE (2), cyclo-cryotherapy (1) or combined ECCE + IOL (1). PK had been combined with intra-capsular cataract extraction (ICCE) in eight cases; overall, 19 patients were aphakic or pseudophakic, 13 following ICCE and six following ECCE. Most PKs (28) were central, mean diameter $8.1 \mathrm{~mm}$, and only one $9 \mathrm{~mm}$ PK was eccentric. Twenty-five were first and four were second PKs. Post-operative steroids were given initially 1-2 hourly dexamethasone $0.1 \%$, as for cataract surgery, and were reduced as indicated by the clinical course. Steroids were maintained indefinitely following tube drainage to avoid the complication of rejection. ${ }^{5}$ Complications, graft survival and visual outcomes were analysed.

Rejection episodes were diagnosed in the presence of anterior chamber cells with graft

Table I Indications for PK prior to further intraocular surgery

\begin{tabular}{|c|c|c|}
\hline & \multicolumn{2}{|c|}{ Total number patients (\%) } \\
\hline & $\begin{array}{c}E C C E( \pm I O L) \\
25(100 \%)\end{array}$ & $\begin{array}{c}\text { Trabeculectomy } \\
29(100 \%)\end{array}$ \\
\hline Keratoconus & $6(24 \%)$ & $1 \quad(3 \%)$ \\
\hline Interstitial keratitis & $6(24 \%)$ & $3(10 \%)$ \\
\hline Corneal perforation & $6(24 \%)$ & $7(24 \%)$ \\
\hline Acanthamoeba & $3(12 \%)$ & $1(3 \%)$ \\
\hline Corneal dystrophies & $2(8 \%)$ & \\
\hline Trauma & $1(4 \%)$ & $4(14 \%)$ \\
\hline HSK & $1(4 \%)$ & $4(14 \%)$ \\
\hline ABK & & $3(10 \%)$ \\
\hline PBK & & $2(7 \%)$ \\
\hline Glaucoma & & \\
\hline complications & & $4(14 \%)$ \\
\hline
\end{tabular}

ABK: aphakic bullous keratopathy

PBK: pseudo-phakic bullous keratopathy. 
endothelial keratic precipitates; a Khodadoust line was not required for the diagnosis. Graft failure was defined as irrecoverable loss of graft clarity.

\section{Results}

\section{Extracapsular Cataract Extraction}

Mean follow-up was 98 months post-PK and 32 months post-ECCE (minimum six months). Kaplan-Meier survival curves ${ }^{6}$ were constructed to demonstrate graft survival. The probability of graft survival after ECCE, with or without lens implantation, was 0.83 after 66 months. The incidence of rejection was $12 \%$ (three cases), all of which occurred during the immediate post-operative period. None resulted in graft failure. One patient with dry eyes developed a suture abscess (Streptococcus pneumoniae) which was successfully treated. One patient required resuturing of the PK after traumatic wound dehissence. Four patients grafted for keratitis developed glaucoma; two after Acanthamoeba keratitis (one required trabeculectomy and a subsequent tube). There were three failures; due to intractable secondary glaucoma (1), gradual decompensation (1) and vascularisation of a $4 \mathrm{~mm}$ paralimbal patch graft (1).

Corrected visual acuity was $6 / 12$ or better in $65 \%$ of cases with clear grafts. Poor vision was attributable to graft failure in two cases. The remainder were due to maculopathy (3), glaucoma (2), retinal vein occlusion (1) and amblyopia (1). The spherical equivalent for patients with IOL was within 3.00 dioptres of emmetropia in $65 \%$ of cases.

\section{Trabeculectomy}

Mean follow-up was 46 months post-PK and 38.5 months post-trabeculectomy. The probability of graft survival after trabeculectomy, including cases which subsequently underwent tube drainage procedures (5) or cyclocryoablation (1), was 0.62 after 45 months. Rejections occurred in $14 \%$ (4) cases, during the post-trabeculectomy period (mean postoperative interval $=5$ months) and resulted in two failures; neither had undergone trabeculectomy within 6 months of PK. Five cases were complicated by infection; three were in failed grafts which developed keratitis (S. pneumoniae and $\mathrm{P}$. aeruginosa). Two cases were successfully treated; one with a suture abscess (S. viridans) and one with endophthalmitis following tube drainage (S. epidermidis).

Eight grafts failed; three due to uncontrolled intra-ocular pressure (IOP), two due to irreversible rejection, two due to recurrent Herpes simplex keratitis (HSK) and one gradual decompensation after tube insertion.

Trabeculectomy alone controlled IOP in ten cases, additional topical therapy was required in 11 (timolol $0.25 \%$ and/or pilocarpine $4 \%$ ) with acetazolamide in three cases. Of the five cases which required tube drainage, four were controlled. Visual outcome for this group was poor; $6 / 12$ or better was achieved in only $35 \%$ of patients with clear grafts. Patients with vision less than $6 / 36(n=6)$ were noted to have glaucomatous disc cupping (4), choroidal haemorrhage (1) and poor ocular surface (1) which probably influenced visual potential.

\section{Discussion}

Graft survival has been considered separately for patients who underwent subsequent cataract extraction or trabeculectomy.

Corneal disease more commonly co-exists with cataract and is frequently managed by combined surgery. Where corneal disease requires $\mathrm{PK}$, a decision should be made with respect to the mangement of any lenticular opacity. Combined surgery has been advocated in preference to two-stage surgery to reduce the risk of late endothelial decompensation. If no significant difference can be established for graft survival between combined and two-stage surgery, the option of deferring cataract surgery can be justified with the advantage of a more predictable refractive outcome.

Grạt survival for 66 triple procedures (PK, ECCE and lens implantation) was reported by Crawford et al (1986) to be $90 \% ;^{7}$ mean post-operative follow-up was 15.8 months. Kirkness reported no graft failures after combined surgery $(n=28)$, with mean follow-up of 28.4 months. $^{8}$ Without detailed knowledge of survival and censorship for these patients, statistical comparison with two-stage surgery cannot be performed. 
The probability of PK survival for patients in this series (post-cataract surgery) was, however, 0.83 (follow-up was 32 months) and appears to compare favourably. PK survival, however, is only a reflection of endothelial function. Endothelial cell counts were not done prospectively although donor material was assessed clinically prior to keratoplasty. Quantitative endothelial assessment of the donor both pre-operatively and post-operatively would be useful further studies. Multiple procedures might be expected to be an adverse prognostic factor in PK survival. Of the 12 patients who underwent two or more procedures, however, only three failed (a similar proportion to the overall failure rate).

Post-operative refraction after combined surgery was found by Crawford et al to be within 2 dioptres of emmetropia in $62 \%$ of cases and by Kirkness et al to be within 3 dioptres of emmetropia in $50 \%$ of cases. Graft sutures had been selectively removed in these patients to reduce astigmatism, but had not been totally removed. It was suggested by Crawford et al that the refractive accuracy of combined surgery (PK + ECCE + IOL) could be improved for individual surgeons by applying a formula derived from regression analysis. ${ }^{7}$ The refractive results for patients reported in this study $(65 \%$ of patients with IOL were within three dioptres of emmetropia) were no better than for combined surgery. Graft sutures had not been removed routinely prior to cataract extraction, however, hence final keratometry was not available when biometry was performed. This should therefore be considered in the future as a means of improving refractive accuracy. Visual outcome was significantly better for the cataract group $(\mathrm{P}=0.025)$. Severe glaucomatous disc cupping occurred more frequently in the trabeculectomy group (28\%) compared with the cataract group (8\%) and the difference in visual outcome was attributable to glaucomatous field loss.

Glaucoma was a risk factor for PK survival $(\mathrm{P}=0.1)$ and occurred in both groups; $4 \%$ (1) of ECCE patients and 14\% (4) in the trabeculectomy group. The probability of maintaining normal intraocular pressure (IOP) by trabeculectomy after PK is less than $50 \%$ after two years. ${ }^{2}$ Since raised IOP results in endothelial dysfunction, patients in whom trabeculectomy fails should be offered tube drainage to protect both optic nerve and graft endothelium.

Combined PK and trabeculectomy is performed less frequently than combined PK and cataract extraction, hence graft survival for combined and two-stage surgery cannot readily be compared for this group. Insler $e t$ al reported one failure in seven cases (mean follow-up 16 months) which underwent combined PK and trabeculectomy. ${ }^{9}$ Foulks reported similar results for PK survival after trabeculectomy for post-keratoplasty glaucoma. ${ }^{10}$ Of five patients who had trabeculectomy, one graft failed, but of 17 who had cyclocryo-ablation, seven grafts failed. Kirkness reported a 0.68 probability of success for controlling intraocular pressure and maintaining graft status after tube drainage. ${ }^{5}$ This was statistically better than for cyclocryoablation $(P=0.05)$. The choice of post-PK glaucoma surgery is therefore important with respect to the risk of failure. Although the survival curves suggest better survival for PKs following subsequent cataract extraction compared with trabeculectomy, the wide confidence limits $(8.9 \%-114 \%)$ indicate this to be an imprecise estimate.

Ocular pathology might be expected to play a role in PK survival. Various pathologies were associated with failure $(n=11)$, but none achieved statistical significance as a risk factor. This may in part be due to the small numbers involved. Coster ${ }^{4}$ found survival was reduced from $76 \%$ to $44 \%$ in eyes which had been inflamed. Management of inflamed eyes influences this risk as shown by Ficker et al for grafting in $\mathrm{HSK}^{11}{ }^{11}$ inflammation was a risk factor in an early cohort $(\mathrm{P}=0.001)$, but not in a later cohort $(\mathrm{P}=0.4)$ managed by ECCE techniques, interrupted suturing and more aggressive topical steroid therapy. Patients with corneal perforation or uncontrolled inflammation, in this series, were managed very aggressively during the acute event with early surgery and intensive post-operative steroids to reduce the risks of peripheral anterior synechiae and early graft rejection. This policy appears to have been effective, with no PK failures in inflamed eyes. 
Rejections were equally common in the cataract extraction and trabeculectomy groups and the risk of failure from graft rejection was similar for both groups $(\mathrm{P}=0.5)$. Rejection episodes were managed early (in the presence of anterior chamber cells, but not requiring a Khodadoust line) with intensive topical steroids and this appears important for PK survival. ${ }^{11}$ Infections occurred in six cases of which $2 / 6$ were suture abscesses. These may be avoidable by prompt removal of loose sutures. Suture infiltrates should be presumed to indicate infection. The suture should be removed for culture in addition to a corneal scrape prior to treatment with intensive topical antibiotics.

We conclude that with modern micro-surgical techniques and the use of intra-ocular visco-elastic fluids, intra-ocular surgery may safely be performed after PK. Rejection caused PK failure in $2 / 54$ cases overall. This compares with failures due to rejection in keratoconus and Fuchs' endothelial dystrophy. Uncontrolled glaucoma was also identified as a risk factor, hence PK survival may indeed depend on glaucoma surgery. Further prospective studies are required to determine whether, following elective graft suture removal, a more predictable refractive outcome may be achieved.

\section{References}

${ }^{1}$ Abbott RL and Forster RK: Clinical specular microscopy and intraocular surgery. Arch Ophthalmol 1979, 97: 1476-9.

${ }^{2}$ Gilvarry AME, Kirkness CM, Steele AD, et al.: The management of post-keratoplasty glaucoma by trabeculectomy. Eye 1989, 3: 713-18.

${ }^{3}$ Schockett SS, Nirankari VS, Lakhanpal V, et al.: Anterior chamber tube shunt to an encircling band in the treatment of neovascular glaucoma and other refractory glaucomas. Ophthalmology 1985, 92: 553-62.

${ }^{4}$ Coster DJ: Mechanisms of corneal graft failure: the erosion of corneal privilege. Eye 1989, 3: 251-62.

${ }^{5}$ Kirkness CM, Ling Y, Rice NSC: The use of silicone drainage tubing to control post-keratoplasty glaucoma. Eye 1988, 2: 583-90.

${ }^{6}$ Kaplan E and Meier P: Non-parametric estimation from incomplete observations.J Am Statis Ass 1958, 53: 457-81.

${ }^{7}$ Crawford GJ, Stulting RD, Waring GO: The triple procedure. Analysis of outcome, refraction and intraocular lens power calculation. Ophthalmology 1986, 93: 817-24.

${ }^{8}$ Kirkness CM, Cheong PY, Steele AD: Penetrating keratoplasty and cataract surgery: the advantages of an extracapsular technique combined with posterior chamber intraocular implantation. Eye 1987, 1: 557-61.

${ }^{9}$ Insler MS, Cooper HD, Kastl PR, et al.: Penetrating keratoplasty with trabeculectomy. Am J Ophthalmol 1985, 100: 593-5.

${ }^{10}$ Foulks GN: Glaucoma associated with penetrating keratoplasty. Ophthalmology 1987, 94: 871-4.

${ }^{11}$ Ficker LA, Kirkness CM, Rice NSC, et al.: The changing management and improved prognosis for corneal grafting in Herpes simplex keratitis. Ophthalmology 1989, 96: 1587-96. 